\title{
Superconductivity in Heavy Fermion Materials
}

\author{
Shailaj Kumar Shrivastava
}

\begin{abstract}
The heavy fermion materials have small superconducting transition temperature and large specific heat corresponding to large effective masses. In these materials the superconductivity co-exists with ferromagnetic or antiferromagnetic order at low temperature. It shows phenomena like magnetic instabilities, quantum critical points (QCP), non-fermi liquid (NFL) and unconventional superconductivity. By comparing the superconducting properties, phase diagram and effect of magnetic field and pressure of heavy fermions based on uranium, cerium, and praseodymium, the basic physics behind pairing mechanism can be imagined. This paper aims to present remarkable findings in superconductivity of various heavy fermion materials.
\end{abstract}

Keywords: Cerium, heavy fermion superconductors, Praseodymium, Unconventional superconductivity, Uranium.

\section{INTRODUCTION}

The heavy fermion materials have rare earth (usually Ce) or actinide element (usually $\mathrm{U}$ ) in which $\mathrm{f}$ electron shell are not fully filled. The interaction of the localized magnetic moments with the momenta and spin of outer shell electrons of the other constituent metal atoms are very strong [1]. The critical temperature of these materials is less than 2.0K.They have magnetic field penetration depth $\lambda$ in excess of several thousand $\AA$ and the values of coherence length $\xi$ are 100$200 \AA$. They are strongly correlated electron systems that exhibit very large effective masses below certain temperature. It was found that the cooper-pairing in these materials arises from the magnetic interactions of the electron spins, rather than by lattice vibrations. In uraniumbased heavy fermions containing a periodic array of uranium ions, the superconductivity co-exists with ferromagnetic or antiferromagnetic order. At quantum critical point (QCP) the magnetic ordering of atoms is destroyed by a small change in pressure. The quantum critical point provides an environment for superconductivity. Near quantum critical points, phase fluctuation with the material expands in both space and time, and these fluctuations help in cooper pair formation. The large value of specific heat $(\mathrm{Cp} / \mathrm{T})$ near transition temperature $\left(\mathrm{T}_{\mathrm{c}}\right)$ indicates that quasi-particles of large masses play an important role in the superconducting pairing.

\section{RESEARCH METODOLOGY}

$\approx 30 \mathrm{~K}$. Superconductivity in $\mathrm{UGe}_{2}$ was observed on the border of ferromagnetism [4]. Fig. 1 shows variation of $\mathrm{P}$ as a function of $\mathrm{T}$ for $\mathrm{UGe}_{2}$ in which the superconducting dome is completely inside the ferromagnetic phase. Here superconductivity coexists with strong ferromagnetism.

Revised Manuscript Received on February 05, 2020.

* Correspondence Author

Shailaj Kumar Shrivastava, Principal, Anugrah Memorial College, Gaya, Bihar, India (A constituent unit of Magadh University, BodhGaya), Email: shailajshri68@yahoo.com

(c) The Authors. Published by Blue Eyes Intelligence Engineering and Sciences Publication (BEIESP). This is an open access article under the CC BY-NC-ND license (http://creativecommons.org/licenses/by-nc-nd/4.0/)

\section{A. Uranium Based Superconductors}

The uranium based superconducting materials exhibit the coexistence of superconductors with a magnetic long range order. In $\mathrm{UPt}_{3}, \mathrm{UBe}_{13}, \mathrm{URu}_{2} \mathrm{Si}_{2}, \mathrm{UPd}_{2} \mathrm{Al}_{3}, \mathrm{UNi}_{2} \mathrm{Al}_{3}$, and $\mathrm{U}_{2} \mathrm{PtC}_{2}$, a transition from to an antiferromagnetic state to a superconducting state takes place. In $\mathrm{UGe}_{2}, \mathrm{URhGe}$ and UCoGe, the superconductivity coexists with the ferromagnetism, resulting in the spin-triplet state of Cooper pairs [2]. In URhGe and UCoGe following changes occurs when magnetic field is applied

$>\mathrm{T}_{\text {curie }}$ is overpowered.

$>$ Ferromagnetic fluctuations are enhanced.

$>$ Superconductivity behavior is enhanced.

In $\mathrm{UPt}_{3}$, the anisotropic order parameter has unconventional symmetry. Superconductivity in hexagonal $\mathrm{UPt}_{3}$ has $\mathrm{T}_{\mathrm{C}} \approx 0.53 \mathrm{~K}$ and below $\mathrm{T}_{\mathrm{c}}$ it exhibit more than one superconducting phase. Below Neel temperature $\left(T_{N}\right)=5 K$, $\mathrm{UPt}_{3}$ is antiferromagnetic with size of magnetic moment $(\mu$ $=0.02 \mu \mathrm{B}$ ). The two superconducting transitions merge into one and antiferromagnetism is destroyed at the same pressure. $\mathrm{UPt}_{3}$ exhibit spin triplet pairing state supporting spin fluctuation mechanism.

$\mathrm{UBe}_{13}$ is a non-fermi liquid (NFL) superconductor with a cubic crystal structure having superconducting transition temperature $\left(T_{c}\right) \approx 0.97 \mathrm{~K}$. The large specific heat (1100 $\mathrm{mJ} / \mathrm{mol}^{2} \mathrm{~K}^{2}$ ) and corresponding high effective mass $(\mathrm{m} * / \mathrm{m} \approx 260)$ shows that the quasi-particles of large masses are involved in superconductivity. The superconducting symmetry has nodal superconducting gap structure due to its unconventional nature.

The body centered tetragonal crystal $\mathrm{URu}_{2} \mathrm{Si}_{2}$ has transition temperature $\left(\mathrm{T}_{\mathrm{c}}\right) \approx 1.5 \mathrm{~K} . \mathrm{URu}_{2} \mathrm{Si}_{2}$ [3] is a multiband superconductor having semi-metallic compensated electronic structure. The hidden order transition takes place at $17.5 \mathrm{~K}$. The order parameter has even parity (spin singlet pairing).

URhGe has $\mathrm{T}_{\text {curie }} \approx 9.5 \mathrm{~K}$ and ferromagnetism appears at ambient pressure followed by superconductivity near $0.3 \mathrm{~K}$ in fields lower than $2 \mathrm{~T}$. The involvement of the same $5 \mathrm{f}$ electrons in these materials shows that the ferromagnetic and superconducting behavior co-exists on the microscopic scale. $\mathrm{UGe}_{2}$ is an orthorhombic crystal having critical temperature $\mathrm{T}_{\mathrm{c}} \approx 0.8 \mathrm{~K}$ at $12 \mathrm{kbar}$. When pressure is applied the ferromagnetism is overpowered at $\mathrm{T}_{\text {curie }}$ 


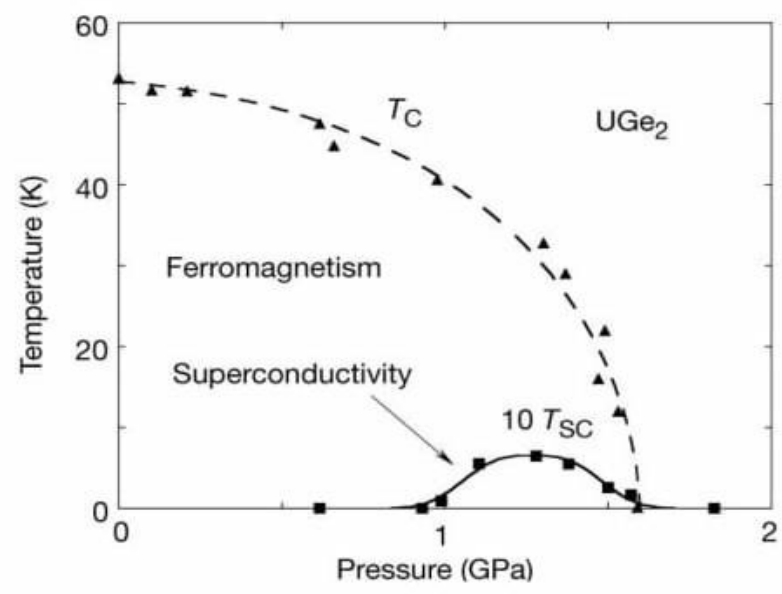

Fig. 1. P-T diagram of UGe2 [5]

$\mathrm{UTe}_{2}$ has superconducting transition temperature $\mathrm{T}_{\mathrm{c}} \approx 1.6 \mathrm{~K}$ at ambient pressure and zero magnetic fields [6]. The upper critical field $\mathrm{H}_{\mathrm{c} 2}(0) \approx 40$ tesla is very large. UTe 2 has body centered orthorhombic structure with lattice constants $\mathrm{a}=4.165 \AA, \quad \mathrm{b}=6.139 \AA$ and $\mathrm{c}=13.979 \AA$. Transition temperature $\left(T_{c}\right)$, crystal structure and magnetism of some uranium based heavy fermion superconductors are given in table I.

Table I: Transition temperature (Tc), crystal structure and magnetism of some uranium based heavy fermion superconductors

\begin{tabular}{|l|c|l|l|}
\hline Material & $\mathrm{T}_{\mathrm{C}} \mathrm{K}$ & $\begin{array}{l}\text { Crystal } \\
\text { Structure }\end{array}$ & Magnetism \\
\hline $\mathrm{UPt}_{3}$ & 0.53 & Hexagonal & Paramagnetic \\
\hline $\mathrm{UBe}_{13}$ & 0.93 & Cubic & Paramagnetic \\
\hline $\mathrm{URu}_{2} \mathrm{Si}_{2}$ & 1.53 & bc tetragonal & $\begin{array}{l}\text { Antiferromagnetic } \\
\left(\mathrm{T}_{\mathrm{N}}=17.5 \mathrm{~K}\right)\end{array}$ \\
\hline $\mathrm{URhGe}$ & 0.25 & orthorhombic & $\begin{array}{l}\text { Ferromagnetic } \\
\left(\mathrm{T}_{\text {curie }}=9.5 \mathrm{~K}\right)\end{array}$ \\
\hline $\mathrm{UPd}_{2} \mathrm{Al}_{3}$ & 2.0 & Hexagonal & $\begin{array}{l}\text { Antiferromagnetic } \\
\left(\mathrm{T}_{\mathrm{N}}=14.5 \mathrm{~K}\right)\end{array}$ \\
\hline $\mathrm{UNi}_{2} \mathrm{Al}_{3}$ & 1.6 & Hexagonal & $\begin{array}{l}\text { Antiferromagnetic } \\
\left(\mathrm{T}_{\mathrm{N}}=4.6 \mathrm{~K}\right)\end{array}$ \\
\hline $\mathrm{UGe}_{2}$ & 0.8 & orthorhombic & $\begin{array}{l}\text { Ferromagnetic } \\
\left(\mathrm{T}_{\text {curie }}=30 \mathrm{~K}\right)\end{array}$ \\
\hline $\mathrm{UTe}_{2}$ & 1.6 & orthorhombic & $\begin{array}{l}\text { Nearly } \\
\text { ferromagnetic }\end{array}$ \\
\hline
\end{tabular}

$\mathrm{UPd}_{2} \mathrm{Al}_{3}$ has transition temperature $\mathrm{T}_{\mathrm{c}} \approx 2.0 \mathrm{~K}$ and having Neel temperature $T_{\mathrm{N}} \approx 14.5 \mathrm{~K}$. When pressure is increased up to $6.5 \mathrm{GPa}$ the Neel temperature $\mathrm{T}_{\mathrm{N}}$ deceases while the onset of superconductivity remains unchanged but above $6.5 \mathrm{GPa}$ the critical temperature starts decreasing. Here pairing is mediated by spin fluctuation.

Hexagonal $\mathrm{UNi}_{2} \mathrm{Al}_{3}$ has transition temperature $\mathrm{T}_{\mathrm{c}} \approx 1.6 \mathrm{~K}$. At $4.5 \mathrm{~K}$, the co-existence of a small magnetic moment with disproportionate spin density wave recommend spin triplet state.

Theoretically, $\mathrm{Hc}_{1} \alpha 1 / \lambda^{2}$, which may leads to anomalously small value of $\mathrm{Hc}_{1}$ and $\mathrm{Hc}_{2} \alpha 1 / \xi^{2}$ which may lead to large value of $\mathrm{Hc}_{2}$ in heavy fermion superconductors. Superconducting parameters of uranium based superconductors are shown in table II.

Table II: Superconducting parameters of uranium based superconductors (Values are taken from the references quoted in the text)

\begin{tabular}{|l|l|l|l|l|}
\hline Materials & $\lambda(\AA)$ & $\xi(\AA)$ & $\begin{array}{l}\mathrm{Hc}_{1}(0) \\
\mathrm{mT}\end{array}$ & $\begin{array}{l}\mathrm{Hc}_{2}(0) \\
\mathrm{T}\end{array}$ \\
\hline $\mathrm{UPt}_{3}$ & $\sim 7000$ & $100-120$ & 3.0 & 2.8 \\
\hline $\mathrm{UBe}_{13}$ & $\sim 8000$ & 100 & 4.6 & 10.1 \\
\hline $\mathrm{UNi}_{2} \mathrm{Al}_{3}$ & $\sim 3000$ & 240 & 1.5 & 1.5 \\
\hline $\mathrm{UPd}_{2} \mathrm{Al}_{3}$ & $\sim 5000$ & 85 & 1.0 & 3.6 \\
\hline $\mathrm{URu}_{2} \mathrm{Si}_{2}$ & $\sim 15000$ & $100-150$ & 1.4 & 3.0 \\
\hline
\end{tabular}

\section{B. Cerium Based Superconductors}

In these materials, there is interplay between two competing mechanisms. Due to strong RKKY interaction in $\mathrm{CeCu}_{6}$ and $\mathrm{CeRu}_{2} \mathrm{Si}_{2}$, it shows collapse of long range magnetism [7]. When pressure is applied to $\mathrm{CeIn}_{3}$, $\mathrm{CeCu}_{2} \mathrm{Si}_{2}, \mathrm{CeCu}_{2} \mathrm{Ge}_{2}, \mathrm{CeRh}_{2} \mathrm{Si}_{2}$ and $\mathrm{CePd}_{2} \mathrm{Si}_{2}$, the Neel temperature $\mathrm{T}_{\mathrm{N}}$ decreases, and a quantum critical point is reached which makes the way for heavy fermion superconductivity to emerge. At quantum critical point a small change in pressure destroy the magnetic ordering of atoms.

In $\mathrm{CeCu}_{2} \mathrm{Si}_{2}$ magnetism and superconductivity do not coexist and has non-Fermi Liquid (NFL) behavior with critical temperature $\left(\mathrm{T}_{\mathrm{c}}\right) \approx 0.7 \mathrm{~K}$ [4]. The superconducting cooper pairs are particle having large effective masses.

The tetragonal crystal CeRhIn ${ }_{5}$ has the layers of $\mathrm{CeIn}_{3}$ separated by layers of $\mathrm{RhIn}_{2}$. At ambient pressure CeRhIn show antiferromagnetism with a Neel temperature $\mathrm{T}_{\mathrm{N}} \approx 3.8$ $\mathrm{K}$. At around 2.3 - 2.5 GPa this antiferromagnetic state is replaced by a superconducting state having transition temperature $\left(\mathrm{T}_{\mathrm{c}}\right) \approx 2.6 \mathrm{~K}$. Fig.2 show the T-P phase diagram of $\mathrm{CeRhIn}_{5}$ in which the antiferromagnetic and superconducting phase co-exists at 2.4GPa. 


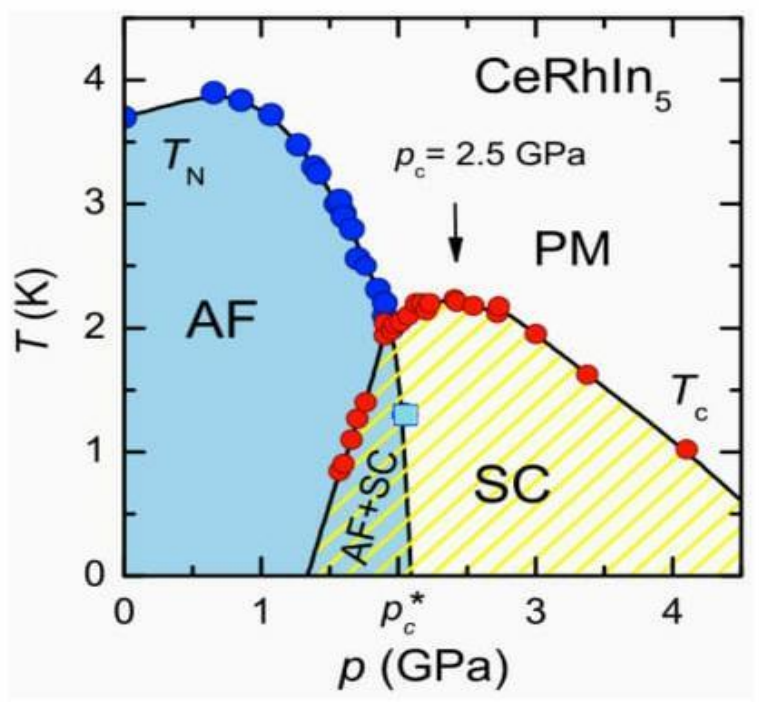

Fig.2. T-P phase diagram of CeRhIn5 [8].

The tetragonal $\mathrm{CeCoIn}_{5}$ has a quasi-2D structure with lattice constants $\mathrm{a}=\mathrm{b}=4.612 \AA$ and $\mathrm{c}=7.551 \AA$. $\mathrm{CeCoIn}_{5}$ has the critical temperature $T_{c}=2.3 \mathrm{~K}$. A specific heat jump of $290 \mathrm{~mJ} / \mathrm{mol}-\mathrm{K}^{2}$ at critical temperature $\left(T_{c}\right)$ has been observed at ambient condition. $\mathrm{CeCoIn}_{5}$ has the layers of $\mathrm{CeIn}_{3}$ separated by layers of $\mathrm{CoIn}_{2}$. The superconductivity in $\mathrm{CeCoIn}_{5}$ arises from magnetic interactions and has line nodes in the order parameter. $\mathrm{CeCoIn}_{5}$ has an unusual pairing symmetry and pairs up in a d-wave state. Under pressure, the fermi liquid behavior is broken in the nonfermi liquid state and superconductivity is realized in CeCoIn 5 .

Tetragonal $\mathrm{CeRh}_{2} \mathrm{Si}_{2}$ show antiferromagnetism at $\mathrm{T}_{\mathrm{N}} \approx 36 \mathrm{~K}$. Below $\mathrm{T}_{\mathrm{N}}=25 \mathrm{~K}$, the antiferromagnetism is replaced by superconducting state at critical pressure $\approx 9$ kbar, superconductivity appears at critical temperature $\left(\mathrm{T}_{\mathrm{c}}\right)$ $\approx 0.4 \mathrm{~K}$. Cerium based heavy fermion superconductors with $\mathrm{T}_{\mathrm{C}}$ and crystal structure are given in table III.

Table III: show cerium based heavy fermion superconductors with Tc and crystal structure.

\begin{tabular}{|l|l|l|}
\hline Materials & $\mathrm{T}_{\mathrm{C}}(\mathrm{K})$ & Crystal Structure \\
\hline $\mathrm{CeCu}_{2} \mathrm{Si}_{2}$ & 0.6 & bc tetragonal \\
\hline $\mathrm{CeRhIn}_{5}$ & 2.6 & tetragonal \\
\hline $\mathrm{CeCoIn}$ & 2.3 & tetragonal \\
\hline $\mathrm{CeIn}_{3}$ & 0.19 & Cubic \\
\hline $\mathrm{CePd}_{2} \mathrm{Si}_{2}$ & 0.43 & bc tetragonal \\
\hline $\mathrm{CeRh}_{2} \mathrm{Si}_{2}$ & 0.3 & bc tetragonal \\
\hline $\mathrm{CePt}_{3} \mathrm{Si}$ & 0.75 & tetragonal \\
\hline
\end{tabular}

At ambient pressure, $\mathrm{CeIn}_{3}$ exhibit antiferromagnetism with temperature $\mathrm{T}_{\mathrm{N}}=10.2 \mathrm{~K}$. When pressure is applied at around $0.19 \mathrm{~K}, \mathrm{~T}_{\mathrm{N}}$ vanishes at a critical pressure of about 26 kbar and below this temperature antiferromagnetism is replaced by a superconducting state. The PressureTemperature phase diagram of $\mathrm{CePd}_{2} \mathrm{Si}_{2}$ and $\mathrm{CeIn}_{3}$ suggests that the superconductivity is magnetically mediated.

Tetragonal $\mathrm{CePt}_{3} \mathrm{Si}$ [9] undergoes a magnetic transition at $\mathrm{T}_{\mathrm{N}}=2.2 \mathrm{~K}$ and a superconductivity transition at $\mathrm{T}_{\mathrm{c}} \approx 0.75 \mathrm{~K}$ at ambient pressure. This material lacks a center of inversion symmetry which shows that it may not be favorable for superconductivity. Higher value of $\mathrm{Hc}_{2}(0) \approx 5 \mathrm{~T}$ indicates the possibility of spin triplet pairing state.

\section{Praseodymium Based Superconductors}

$\mathrm{PrOs}_{4} \mathrm{Sb}_{12}$ is filled skutterudites heavy fermion compound having superconducting critical temperature $\mathrm{T}_{\mathrm{C}} \approx 1.85 \mathrm{~K}$ with broken time reversal symmetry [10]. It consists of more than one superconducting phases. The field induced antiferroquadrupolar order phase just above the upper critical field indicates that electric quadrupole fluctuations play an important role in the superconducting mechanism.

$\mathrm{PrV}_{2} \mathrm{Al}_{20}$ have the nonmagnetic cubic $\Gamma_{3}$ doublet ground state. It exhibits superconductivity at transition temperature $\mathrm{T}_{\mathrm{c}} \approx 50 \mathrm{mK}$ under ambient pressure. The large $\gamma \approx 300 \mathrm{~m} \mathrm{Jmol}^{-1} \mathrm{~K}^{-2}$ and corresponding large $\mathrm{m} * / \mathrm{m}$ $\approx 140$ indicates the role of orbital fluctuation of the $\mathrm{f}$ electrons at ambient pressure. The gapless mode associated with quadrupolar ordering is indicated in cubic temperature dependence of specific heat capacity.

Cubic $\mathrm{PrTi}_{2} \mathrm{Al}_{20}$ exhibits quadrupolar ordered state and has strong interaction between $4 \mathrm{f}$ and outer shell electrons. It has transition temperature $T_{c}=200 \mathrm{mK}$ in the non magnetic ferro-quadrupolar state. Praseodymium based heavy fermion superconductors with $T_{c}$ and crystal structure is given in table IV.

\section{Table IV: Superconducting transition temperature (Tc) and crystal structure of praseodymium based heavy fermion superconductors.}

\begin{tabular}{|l|l|l|}
\hline Materials & $T_{c}(\mathrm{~K})$ & Crystal Structure \\
\hline $\mathrm{PrOs}_{4} \mathrm{Sb}_{12}$ & 1.85 & Skutterudite \\
\hline $\mathrm{PrV}_{2} \mathrm{Al}_{20}$ & 0.05 & Cubic \\
\hline $\mathrm{PrTi}_{2} \mathrm{Al}_{20}$ & 0.2 & Cubic \\
\hline
\end{tabular}

\section{RESULT ANALYSIS}

Heavy fermion superconductors are highly anisotropic and have close relationship with the possible pairing mechanism. Parity pairing state of heavy fermion materials are shown in Table V.

Table V: Parity pairing state of heavy fermion materials

\begin{tabular}{|l|l|}
\hline Materials & Parity pairing state \\
\hline $\mathrm{CeCoIn}$ & Even \\
\hline $\mathrm{CeCu}_{2} \mathrm{Si}_{2}$ & Even \\
\hline $\mathrm{UPd}_{2} \mathrm{Al}_{3}$ & Even \\
\hline $\mathrm{URu}_{2} \mathrm{Si}_{2}$ & Even \\
\hline $\mathrm{UPt}_{3}$ & Odd \\
\hline $\mathrm{UNi}_{2} \mathrm{Al}_{3}$ & Odd \\
\hline
\end{tabular}

In $\mathrm{CeCu}_{2} \mathrm{Si}_{2}, \mathrm{CeIrIn}_{5}$ [11], $\mathrm{CeCoIn}_{5}$ and $\mathrm{UPd}_{2} \mathrm{Al}_{3}$ the order parameter is of even parity and the NMR Knight Shift decreases below $\mathrm{T}_{\mathrm{c}}$. In $\mathrm{UPt}_{3}$ and $\mathrm{UNi}_{2} \mathrm{Al}_{3}$, odd parity pairing state is observed with no reduction in the NMR Knight Shift. Thus, NMR Knight Shift result classifies the heavy fermion superconductors into either even or odd parity pairing. The non-centrosymmetric crystals $\mathrm{CePt}_{3} \mathrm{Si}$ classify superconductivity with mixing of even and odd parity. The spin fluctuation model predicts that the ferromagnetic interactions should lead to odd parity pairing state while the antiferromagnetic spin fluctuations should lead to even parity pairing state.
Retrieval Number: C5162029320/2020@BEIESP DOI: 10.35940/ijeat.C5162.029320 Journal Website: www.ijeat.org 
As $\gamma \alpha \mathrm{m}^{*}$, cooper pair are particles consisting of large mass. Table VI show the Sommerfeld coefficient of specific heat and effective mass of uranium based superconductors.

Table VI: Sommerfeld coefficient $(\gamma)$ and effective mass of uranium based heavy fermion superconductors.

\begin{tabular}{|l|l|l|}
\hline Materials & $\gamma\left[\mathrm{m} \mathrm{Jmol}^{-1} \mathrm{~K}^{-2}\right]$ & $\mathrm{m} / \mathrm{m}$ \\
\hline $\mathrm{URu}_{2} \mathrm{Si}_{2}$ & 65 & 140 \\
\hline $\mathrm{UPd}_{2} \mathrm{Al}_{3}$ & $145-210$ & 66 \\
\hline $\mathrm{UNi}_{2} \mathrm{Al}_{3}$ & 120 & 48 \\
\hline $\mathrm{UPt}_{3}$ & 450 & 180 \\
\hline $\mathrm{UBe}_{13}$ & 1100 & 260 \\
\hline
\end{tabular}

In $\mathrm{CeIn}_{3}$ and $\mathrm{CePd}_{2} \mathrm{Si}_{2}$ antiferromagnetism is replaced by superconductivity at quantum critical point under applied pressure. In $\mathrm{UPt}_{3}, \mathrm{URu}_{2} \mathrm{Si}_{2}, \mathrm{UPd}_{2} \mathrm{Al}_{3}$ and $\mathrm{UNi}_{2} \mathrm{Al}_{3}$ magnetism and superconductivity co-exist on microscopic scale. In $\mathrm{UGe}_{2}$, URhGe superconductivity appears inside a ferromagnetic phase near quantum critical point. The electron-electron interactions that lead to the Kondo effect dominating the RKKY interaction lead to a lot of quantum critical points and magnetic phase transitions. The presence of a superconducting dome near antiferromagnetic quantum critical point indicates that the quantum critical point (QCP) has its importance in pairing mechanism.

The presence of two superconducting transitions in $\mathrm{PrOs}_{4} \mathrm{Sb}_{12}$ suggests for a homogeneous co-existence of two superconducting order parameters. Here quantum quadrupole fluctuations and the gap structure are vital for understanding the pairing mechanism of superconductors.

\section{CONCLUSION}

The complicated crystal structures, unusual magnetic and superconducting transition, co-existence of superconductivity and antiferromagnetism, multiple superconducting phases, appearance of superconductivity at quantum critical point and the gap structure indicate that pairing mechanism is still a difficult problem in heavy fermion superconductors.

\section{REFERENCES}

1. Piers Coleman. "Heavy Fermions: Electrons at the Edge of Magnetism". Handbook of Magnetism and Advanced Magnetic Materials. In Helmut Kronmuller and Stuart Parkin (Eds), Vol. 1, 95148.John Wiley \&Sons Ltd.ISBN:978-0-470-02217-7

2. D.Aoki, K.William, I.Sheikin. "Heavy fermions in high magnetic fields". Comptes rendus-physique", 14, 1, 53-77, 2013

3. N.Shah, P. Chandra, P. Coleman and J.A. Mydosh" Hidden order in $\mathrm{URu}_{2} \mathrm{Si}_{2}$ " Phys. Rev. B,61(564),2000

4. B.D.White, J.D. Thompson, M.B. Maple. "Unconventional superconductivity in heavy-fermion compounds".Physica C: superconductivity and its applications.514, 246-278, 2015

5. S.S. Saxena, P. Agarwal, K. Ahilan, et al., "Superconductivity on the border of itinerant-electron ferromagnetism in $\mathrm{UGe}_{2}$ " . Nature, 406(6796), 587-92, 2000

6. Sheng Ran, Chris Eckberg, Quing-ping-Ding, Yuji Furukawa et al. "Nearly ferromagnetic spin-triplet superconductivity". Science, 365(6454) 684, 2019

7. Y.Onuki, A.Hsegawa. "Fermi surfaces of intermetallic compounds". Vol. 20 Elsevier, Amsterdam, p49, Chapter 135, 1995

8. G.Knebel, D.Aoki and J. Flouquet. "Antiferromagnetism and superconductivity in cerium based heavy fermion compounds", Comptes Rendus Physique, 12(5-6), 542-566, 2011

9. K.V. Samokhin, E.S. Zijlstra, S.K. Bose. "CePt ${ }_{3} \mathrm{Si}$ : an unconventional superconductor without inversion centre”. Phys. Rev.B, 69, 094514, 2004.

10. M.B. Maple, N.A. Frederick P.-C. Ho, W.M. Yuhasz, T. Yanagisawa. "Unconventional superconductivity and heavy fermion behavior in $\mathrm{PrOs}_{4} \mathrm{Sb}_{12}$ ". J. Supercond. And Novel Magnetism19, 3-5,299-315, 2006 years of teaching and research experience with more than fifty research papers publications in various Journals and conferences. He got several awards including 'Young Research Award' at IUMRS-ICA-98 held at IISc Bangalore.
Shailaj Kumar Shrivastava, worked as Research fellow at National Physical Laboratory, New Delhi and obtained his Ph.D. degree in Physical Science from Delhi University in 2002. His research interest is directed towards superconductivity, thin films and 